\title{
Development of Novel Optical Fiber System for Cathodoluminescence Detection in High Voltage Transmission Electron Microscope
}

\author{
Kenichiro Furumoto ${ }^{1, *}$, Tetsuo Tanabe ${ }^{1}$, Naoji Yamamoto ${ }^{1}$, \\ Takeshi Daio ${ }^{2}$, Syo Matsumura ${ }^{3}$ and Kazuhiro Yasuda ${ }^{3}$ \\ ${ }^{1}$ Interdisciplinary Graduate School of Engineering Science, Kyushu University, Kasuga 816-8580, Japan \\ ${ }^{2}$ High Voltage Electron Microscopy Laboratory, Kyushu University, Fukuoka 819-0395, Japan \\ ${ }^{3}$ Department of Applied Quantum Physics and Nuclear Engineering, Kyushu University, Fukuoka 819-0395, Japan
}

\begin{abstract}
We have installed a new system to observe cathodoluminescence (CL) in a high voltage transmission electron microscope (HVTEM). The system is constructed of a ball lens for collection of CL, an optical fiber and a multi-channel optical detector. The system can be operated without any disturbance of TEM observation. The system was proved to be very useful to observe CL in HVTEM and will be a powerful tool to investigate production mechanisms of luminescence centers by observing CL changes with incident electron energy and flux (displacement damage effect), and temperature, together with simultaneous TEM observations of microstructure changes.

[doi:10.2320/matertrans.M2013025]
\end{abstract}

(Received January 21, 2013; Accepted March 4, 2013; Published April 25, 2013)

Keywords: high voltage transmission electron microscope, cathodoluminescence detection system, in-situ observation, aluminum oxide

\section{Introduction}

We have developed a new equipment to observe cathodoluminescence (CL) in a high voltage transmission electron microscope (HVTEM). CL in SEM or TEM has been used as a useful tool to study correlations of optical properties of semiconductors and insulators with their microstructure. ${ }^{1-3)}$ Fortunately or unfortunately, electron energies ordinary used in SEM and TEM are below $\sim 200 \mathrm{keV}$, which is not enough to induce elastic displacements of atoms and ensure no-micro-structure change during measurements of CL spectra. If we can use higher energy electron beam, in-situ observation of luminescence change accompanied with microstructure change caused by atomic displacement becomes possible. In addition, observation with changing temperature would help to investigate dynamic processes of radiation damage due to atomic displacement effects. This motivated us to equip a CL detection system in HVTEM.

\section{Experimental Setup}

Figure 1 shows a schematic illustration of the present CL detection system equipped into HVTEM (JEM-1300NEF, JEOL Ltd.) at Research Laboratory for High Voltage Electron Microscopy of Kyushu University. An optical fiber system was sheathed in a stainless tube and directly inserted through a small hole with $2 \mathrm{~mm}$ in diameter at an angle of 46 degrees from the electron beam without any disturbance of TEM observation. Therefore all equipments in HVTEM, such as a temperature variable sample holder and other analyzing tools can be used during the collection of CL signals. The optical fiber is made of UV grade $\mathrm{SiO}_{2}$ with a core diameter of $600 \mu \mathrm{m}$. A ball lens of $1 \mathrm{~mm}$ in diameter on the front head of the fiber enhances detection efficiency. The fiber system is movable to be inserted only at measurements of CL signals.

${ }^{*}$ Graduate Student, Kyushu University. Corresponding author, E-mail: furumoto@aees.kyushu-u.ac.jp

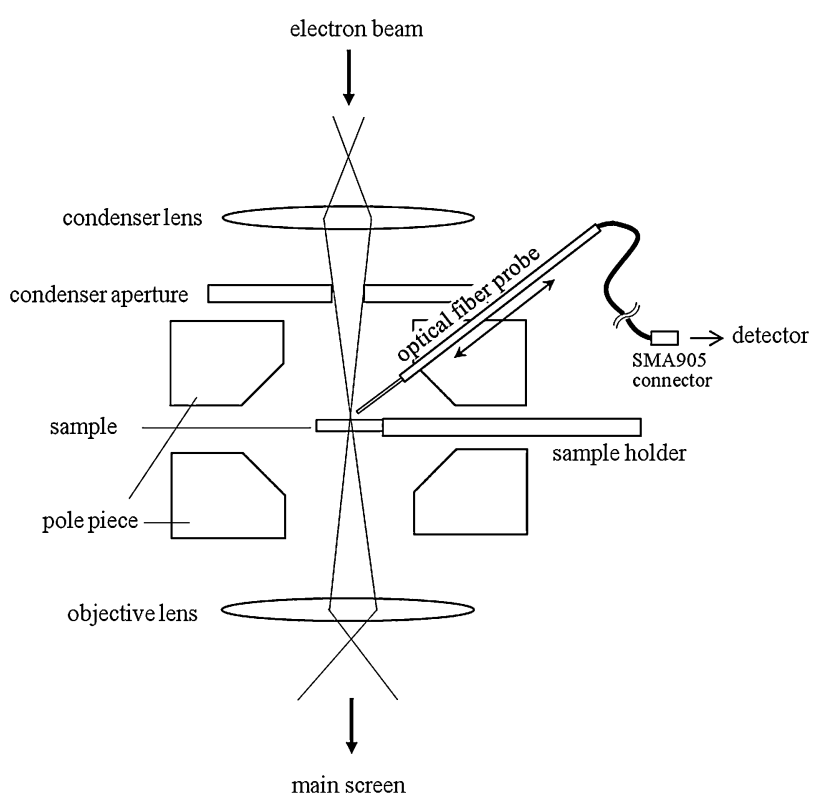

Fig. 1 Schematic illustration of the optical fiber system for TEM-CL detection.

$\mathrm{CL}$ is lead to an external multichannel optical spectrometer (PMA-12, Hamamatsu Photonics) through a SMA905 optical fiber connector.

\section{Result}

Figure 2 shows examples of observed spectra of TEM-CL for a single crystal $\alpha-\mathrm{Al}_{2} \mathrm{O}_{3}$ (sapphire, Furuuchi Chemical Corp.) with the shape of a TEM disk of $3 \mathrm{~mm}$ in diameter and $\sim 150 \mu \mathrm{m}$ in thickness induced by irradiation of a $1.25 \mathrm{MeV}$ electron beam with a diameter of $10 \mu \mathrm{m}$ and flux of $1.3 \times 10^{22}$ electrons $/ \mathrm{m}^{2} \cdot \mathrm{s}$. The observed TEM-CL spectrum is very fine owing to the stainless sheath, which suppressed luminescence of the optical system induced by reflected or scattered electrons in a TEM system previously reported. ${ }^{4)}$ 


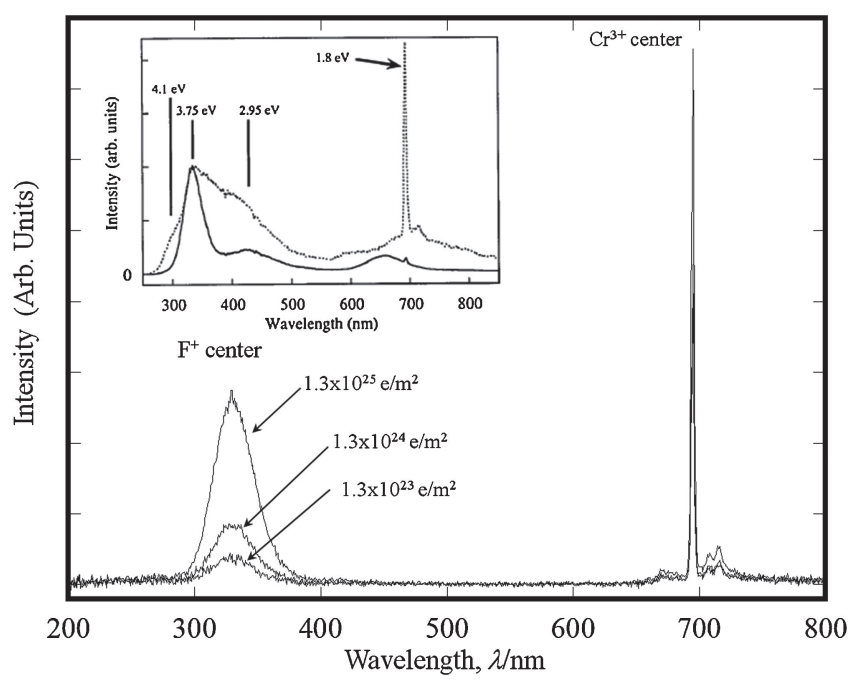

Fig. 2 TEM-CL spectra of $\alpha-\mathrm{Al}_{2} \mathrm{O}_{3}$ measured at $R T$ under irradiation of $1.25 \mathrm{MeV}$ electrons with a flux of $1.3 \times 10^{22}$ electrons $/ \mathrm{m}^{2} \cdot \mathrm{s}$. Inset in the figure shows a typical CL spectrum of $\alpha-\mathrm{Al}_{2} \mathrm{O}_{3}{ }^{5}$ ) for a comparison.

For comparison, a CL spectrum of $\alpha-\mathrm{Al}_{2} \mathrm{O}_{3}$ reported by $\mathrm{P}$. Jonnard et al. ${ }^{5)}$ was shown in the inset of the figure in which the solid and dashed lines were induced by $4 \mathrm{keV}$ electron beam irradiation with electron fluxes of $9.4 \times 10^{19}$ and $9.4 \times 10^{18}$ electrons $/ \mathrm{m}^{2} \cdot \mathrm{s}$, respectively. The present $\mathrm{CL}$ spectrum is composed of intense line emission at $690 \mathrm{~nm}$ $(1.8 \mathrm{eV})$ accompanying a few satellite peaks and a broad band emission peaked at $330 \mathrm{~nm}(3.8 \mathrm{eV})$. In present work, we used thick specimen $(\sim 150 \mu \mathrm{m})$ for the confirmation of $\mathrm{CL}$ detection. Considering the detection limit of the present system, the specimen thickness can be reduced by a factor of $1 / 100$ (assuming the luminescence intensity is proportional to the thickness). Utilization of high-voltage TEM would allow to use a rather thick sample for simultaneous observations of TEM and luminescence.

The intense line emission is assigned as the emission of $\mathrm{Cr}^{3+}$ included as impurities. It is known that the wave length of the line emission and its satellite structures are varied reflecting local atomic arrangements surrounding $\mathrm{Cr}$ ions. $\left.{ }^{6}\right)$ Broad band emission at $330 \mathrm{~nm}$ is attributed to the emission of $\mathrm{F}^{+}$center, which is an oxygen vacancy trapping one electron. In the observed spectra, no emission of $\mathrm{F}$ center (oxygen vacancy trapping two electrons) at around $410 \mathrm{~nm}$ $(3.0 \mathrm{eV})$ appeared. ${ }^{5)}$ In general, $\mathrm{CL}$ spectra for $\mathrm{Al}_{2} \mathrm{O}_{3}$ show double band emissions attributed to $\mathrm{F}$ and $\mathrm{F}^{+}$centers. Such double band emissions, in particular, the intensity ratio of $\mathrm{F}^{+} / \mathrm{F}$ emission changed with the flux and the fluence as shown in the inset of Fig. 2. With increasing the flux and/or the fluence, the intensity ratio of $\mathrm{F}^{+} / \mathrm{F}$ increases. ${ }^{7)}$ It seems that the present irradiation flux of $1.3 \times 10^{22}$ electrons $/ \mathrm{m}^{2} \cdot \mathrm{s}$, was too high to appear the $\mathrm{F}$ center emission.

The benefit of the HVTEM-CL appears in the observation of dynamic changes in the spectra. Figure 3 shows time sequences of the $\mathrm{F}^{+}$emission intensity. At earlier stage of the irradiation (or at lower fluences), the $\mathrm{F}^{+}$emission intensity increased nearly linearly with increasing irradiation time (fluence), while it saturated over the fluence of $\sim 1.5 \times 10^{25}$ electrons $/ \mathrm{m}^{2}$. The linear increase with the fluence can be correlated to the linear increase of the number of oxygen

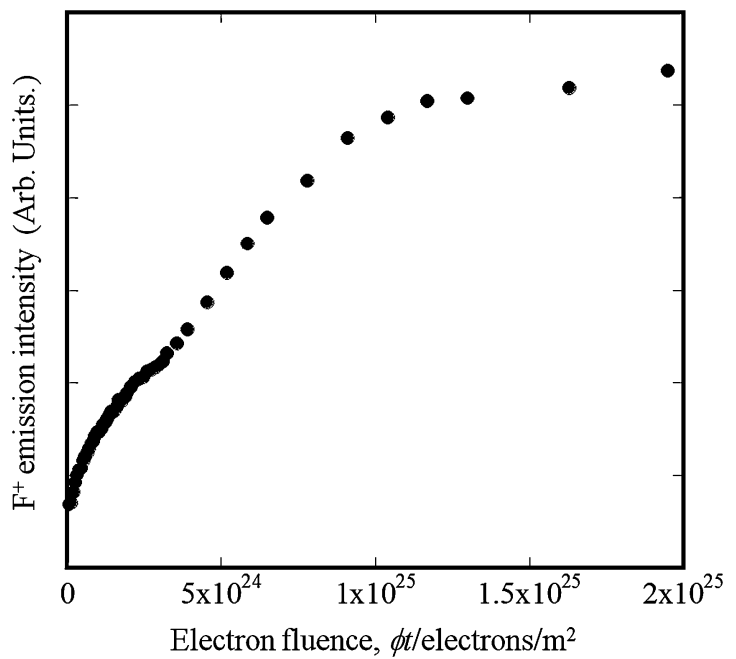

Fig. 3 Electron fluence dependence of $\mathrm{F}^{+}$emission intensity at $R T$.

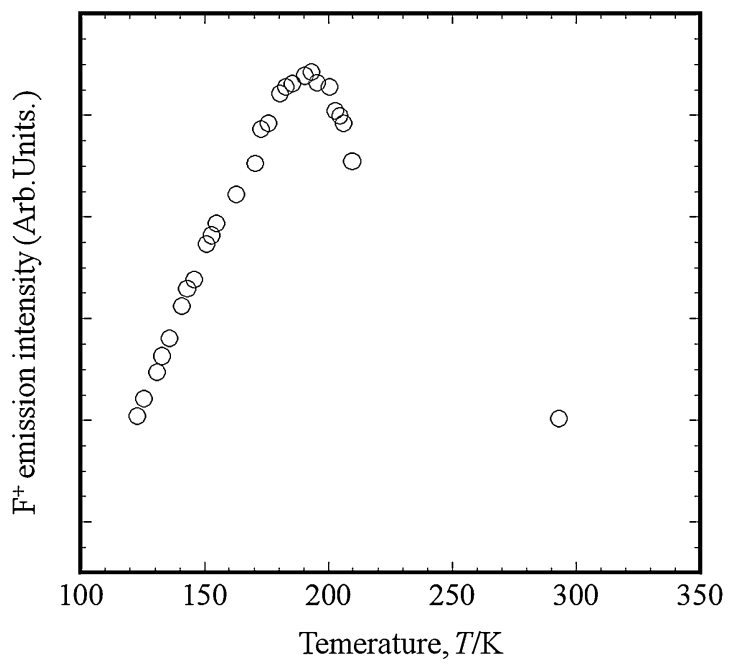

Fig. 4 Temperature dependence of saturated $\mathrm{F}^{+}$emission intensity ranging from $R T$ to $120 \mathrm{~K}$.

vacancies as the source of the luminescence centers produced by an atomic displacement effect of the high-voltage electron irradiation. The appearance of small deviation from the linearity at fluencies around $4 \times 10^{24}$ electrons $/ \mathrm{m}^{2}$ suggests the existence of some additional mechanisms for the increase of emission intensity, which will be investigated in future. Meanwhile, the saturation of the intensity suggests the balance between production of oxygen vacancies and their annihilation by recombination of vacancies and interstitials, and transformation to different defects, such as clustering to multiple vacancies, dislocations and so on.

Using the temperature variable sample holder, the temperature dependence of HVTEM-CL intensity was successfully measured. We observed the change of saturated $\mathrm{F}^{+}$emission intensity ranging from $120 \mathrm{~K}$ to $R T$. After $4.0 \times 10^{25}$ electrons $/ \mathrm{m}^{2}$ irradiation at $R T$, then we cooled a specimen with liquid nitrogen, and measured CL intensity change caused by cooling under continuous irradiation. The result was shown in Fig. 4. The intensity increased with decreasing temperature down to around $190 \mathrm{~K}$ to show a maximum value, and then decreased. Similar temperature dependence of $\mathrm{F}^{+}$ 
emission intensity was previously reported. ${ }^{8)}$ Such observed temperature dependence of the $\mathrm{F}^{+}$emission intensity might not be caused by a simple origin. Since trapped electrons at $\mathrm{F}^{+}$centers are relaxed by either radiative or non-radiative transition, and the non-radiative transition is thermal-activated process, the ratio of radiative and non-radiative transitions is varied with temperature. In addition, saturated concentration of $\mathrm{F}^{+}$centers must be changed with temperature. Detailed investigation of the temperature dependence of the luminescence intensity with and without displacement damage will give useful information on the production or annihilation mechanism of the luminescence centers.

\section{Conclusion}

The newly developed HVTEM-CL detection system is proved to be a powerful tool to investigate production mechanisms of luminescence centers by observing CL changes with incident energy and flux (displacement effect), and temperature, together with simultaneous TEM observations of microstructure changes.

\section{Acknowledgements}

The authors are grateful to Drs. Shigemasa Ohta and Mitsuaki Ohsaki of JEOL Ltd. and their technical assistance to develop the HVTEM-CL equipment. This work was supported by HVEM Laboratory of Kyushu University under a program of HVEM-project.

\section{REFERENCES}

1) S. J. Pennycook: Ultramicroscopy 7 (1981) 99-104.

2) Y. Ohno and S. Takeda: Rev. Sci. Instrum. 66 (1995) 4866.

3) T. Mitsui, N. Yamamoto, T. Tadokoro and S. Ohta: J. Appl. Phys. 80 (1996) 6972.

4) T. Tanabe, S. Muto and S. Tohtake: J. Electron. Microsc. 51 (2002) 311313.

5) P. Jonnard, C. Bonnelle, G. Blaise, G. Rémond and C. Roques-Carmes: J. Appl. Phys. 88 (2000) 6413.

6) D. Lapraz, P. Iaccon, D. Daville and B. Guilho: Phys. Status Solidi A Appl. Mater. Sci. 126 (1991) 521-531.

7) A. Moroño and E. R. Hodgson: J. Nucl. Mater. 249 (1997) 128-132.

8) D. W. Cooke: J. Appl. Phys. 55 (1984) 3437-3440. 